\title{
Antiviral effect of arginine against herpes simplex virus type 1
}

\author{
TAKESHI NAITO ${ }^{1}$, HIROSHI IRIE ${ }^{2}$, KAZUKO TSUJIMOTO $^{3}$, \\ KEIKO IKEDA ${ }^{4}$, TSUTOMU ARAKAWA ${ }^{5}$ and A. HAJIME KOYAMA ${ }^{3}$
}

\author{
${ }^{1}$ Department of Ophthalmology, Institute of Health Biosciences, The University of Tokushima Graduate School, \\ Tokushima 770-8503; ${ }^{2}$ Department of Anatomy, Teikyo University School of Medicine, Kaga-Itabashi 173-8605; \\ ${ }^{3}$ Division of Virology, Department of Cellular and Molecular Medicine, Wakayama Medical University Graduate School \\ of Medicine, Wakayama 641-8509; ${ }^{4}$ School of Health and Nursing Science, Wakayama Medical University, \\ Wakayama 641-0011, Japan; ${ }^{5}$ Alliance Protein Laboratories, Thousand Oaks, CA 91360, USA
}

Received October 1, 2008; Accepted November 26, 2008

\section{DOI: 10.3892/ijmm_00000156}

\begin{abstract}
We investigated the effects of arginine on the multiplication of herpes simplex virus type 1 (HSV-1) and the potential of arginine as an antiherpetic agent. Arginine suppressed the growth of HSV-1 concentration-dependently. Inhibition of HSV-1 by arginine leveled off at $50-60 \mathrm{mM}$, although the higher concentration was not suitable as an antiviral agent due to cytotoxicity. 'Time of addition' experiments revealed that arginine was particularly effective when added within $6 \mathrm{~h}$ post-infection (h p.i.), suggesting that the reagent sensitive step is in the early stages of the infection. A one-step growth curve of HSV-1 in the presence of $30 \mathrm{mM}$ arginine revealed that: i) the latent period was significantly extended, ii) the rate of formation of progeny infectious virus decreased and iii) the final yield of progeny virus decreased to $1 \%$. The addition of arginine at $8 \mathrm{~h} \mathrm{p.i.,} \mathrm{after} \mathrm{the} \mathrm{completion} \mathrm{of} \mathrm{viral}$ DNA replication in the virus multiplication, allowed the normal formation of progeny virus in the subsequent $4 \mathrm{~h}$, confirming that arginine does not directly interfere with the formation of progeny infectious virus. In addition, arginine also inhibits several RNA viruses.
\end{abstract}

\section{Introduction.}

Certain components of foods and drinks show a variety of biological activities and have been adopted in traditional

Correspondence to: Dr Takeshi Naito, Department of Ophthalmology, Institute of Health Biosciences, The University of Tokushima Graduate School, Tokushima 770-8503, Japan

E-mail: naito@clin.med.tokushima-u.ac.jp

Dr A. Hajime Koyama, Division of Virology, Department of Cellular and Molecular Medicine, Wakayama Medical University Graduate School of Medicine, 811-1 Kimiidera, Wakayama 6418509, Japan

E-mail: koyama@wakayama-med.ac.jp

Key words: antiviral, arginine, herpes simplex virus, influenza virus, vesicular stomatitis virus medicine as well as used in healthcare beverages worldwide. They often show an antimicrobial effect (1) and have been used as potential sources for new chemotherapeutic drugs against various pathogenic microbes (2). Arginine is one of the twenty natural amino acids and is found in a wide variety of foods and drinks. This amino acid has a unique long carboncontaining side chain and a complex guanidinium group at the end. Previously, we observed that arginine inactivates a variety of viruses, depending on concentration, incubation $\mathrm{pH}$ and temperature (3-5). Arginine showed a synergistic effect on virus inactivation with low $\mathrm{pH}$ or increased temperature. Virus inactivation is a critical step for biopharmaceutical production (6), indicating the usefulness of arginine for virus clearance, although the mechanism of enhanced virus inactivation by arginine is unclear.

During the course of characterization of virus inactivation by arginine, we observed that arginine affects the multiplication of herpes simplex virus (HSV-1) at relatively low concentrations, far below the concentration at which arginine exerts virucidal activity $(3,4)$. Considering the uniqueness of arginine among amino acids, we further characterized the effect of arginine on the multiplication of DNA and RNA viruses with a prospect that arginine may be effective as an antiviral agent againist various virus infections. A particular focus was given to HSV-1, as this virus causes serious systemic or topical diseases, such as genital ulcerative diseases and ocular herpetic stromal keratitis.

\section{Materials and methods}

Cells and viruses. HEp-2, MDCK and Vero cells were grown in Eagle's minimum essential medium (MEM) containing 5\% fetal bovine serum (FBS). Herpes simplex virus type 1, strain F (HSV-1), influenza virus A/Aichi/68 $\left(\mathrm{H}_{3} \mathrm{~N}_{2}\right)$, poliovirus type 1, Sabin vaccine strain, and vesicular stomatitis virus, New Jersey strain (VSV), were used throughout the experiments. HSV-1, poliovirus and VSV were propagated in Vero cells cultured in MEM supplemented with $0.5 \%$ FBS. Influenza virus was propagated in MDCK cells cultured in MEM supplemented with $0.1 \%$ bovine serum albumin (BSA) and acetylated trypsin $(4 \mu \mathrm{g} / \mathrm{ml})$. These viruses were stored at $-80^{\circ} \mathrm{C}$ until use. Poliovirus-infected cells were incubated at $35.5^{\circ} \mathrm{C}$, as this 
virus is a temperature-sensitive mutant strain. The amount of virus was measured by a plaque assay on Vero or MDCK cells as described previously (7-10).

Reagents. L-arginine hydrochloride (simply described as arginine) was obtained from Ajinomoto Co. Inc. The $\mathrm{pH}$ of the aqueous solution containing arginine was adjusted to 7.0 with $\mathrm{HCl}$ or $\mathrm{NaOH}$ depending on the batch of arginine. The $\mathrm{pH}$ meter was routinely calibrated using $\mathrm{pH}$ calibration standards.

Virus yields in the presence of arginine. The effect of arginine on the multiplication of the virus was examined as follows. Monolayered cells in 35-mm dishes were infected with the virus at an indicated multiplicity of infection (MOI). The infected cells were further incubated at $37^{\circ} \mathrm{C}$ for the indicated period in the serum-free MEM containing $0.1 \% \mathrm{BSA}$ and the indicated concentrations of arginine. For the influenza virus, acetylated trypsin $(4 \mu \mathrm{g} / \mathrm{ml})$ was added to the culture medium. At the indicated time, the amounts of progeny virus in the infected cultures were determined as described previously (7-10). Briefly, for HSV-1 and poliovirus, after two or three cycles of freezing and thawing the infected cells along with the culture media, the number of infectious viruses in the lysate was measured by a plaque assay on Vero cells. For influenza virus and VSV, an aliquot of the culture fluid was harvested to determine the number of progeny viruses by a plaque assay on MDCK and Vero cells, respectively.

Determination of cytopathic effects and cell death. The cytopathic effects (CPE) were determined by microscopic observation of the cells; approximate amounts of rounded cells on monolayers were estimated with a phase-contrast microscope. The extent of cell death in the cultures was determined as follows. The monolayered cells were trypsinized to obtain single-cell suspension. After the addition of MEM containing $5 \%$ FBS to the suspension to neutralize trypsin and stabilize the cells, the numbers of the living and dead cells were measured by a dye-exclusion method with trypan blue.

\section{Results}

The effects of arginine on virus multiplication in the HSV-1infected HEp- 2 cells were examined first. Fig. 1 shows the relative virus yield in the presence of arginine at different concentrations. Arginine suppressed the virus yield concentration-dependently, reaching a maximum antiviral activity at $50 \mathrm{mM}$. It is evident in Fig. 1 that arginine is effective as an antiviral agent at relatively low concentrations, with a 1-log, $2-\log$ and 3-log reduction of the relative virus yield at $\sim 25,35$ and $50 \mathrm{mM}$. Although these arginine concentrations are not within a physiological level, the concentration seems to be within a tolerable range as described later. The antiviral activity of arginine described above has no relation to the previously observed direct virus inactivation activity of arginine because there was no significant virus inactivation when HSV-1 was incubated with arginine up to $0.6 \mathrm{M}$ at $37^{\circ} \mathrm{C}$ for $20 \mathrm{~min}$ (data not shown). Thus, antiviral effects of arginine are mediated, not by virucidal activity but by other factors.

Next, we examined the cytotoxicity of arginine on HEp-2 cells because arginine showed a notable CPE on HEp-2 cells.

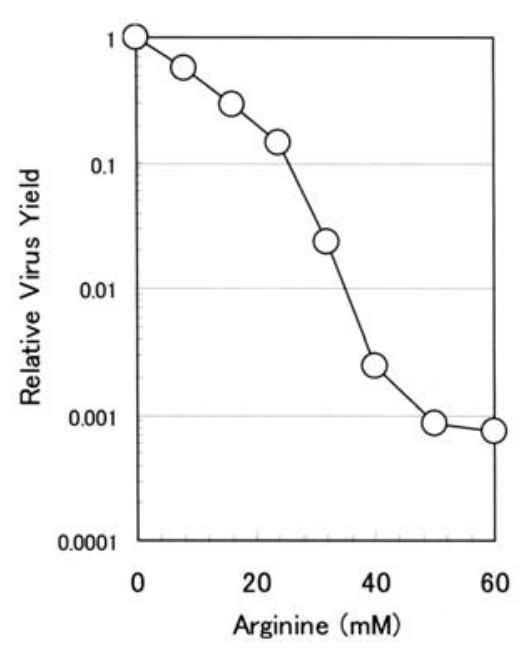

Figure 1. Effect of arginine on the yield of HSV-1 in HEp-2 cells. Confluent monolayers of HEp-2 cells were infected with HSV-1 at an MOI of 10. The infected cells were incubated at $37^{\circ} \mathrm{C}$ for $\sim 27 \mathrm{~h}$ in MEM containing $0.1 \%$ BSA and the indicated concentrations of arginine. The amounts of total progeny viruses were determined as described in Materials and methods and were normalized to the virus yield in the absence of the reagent.

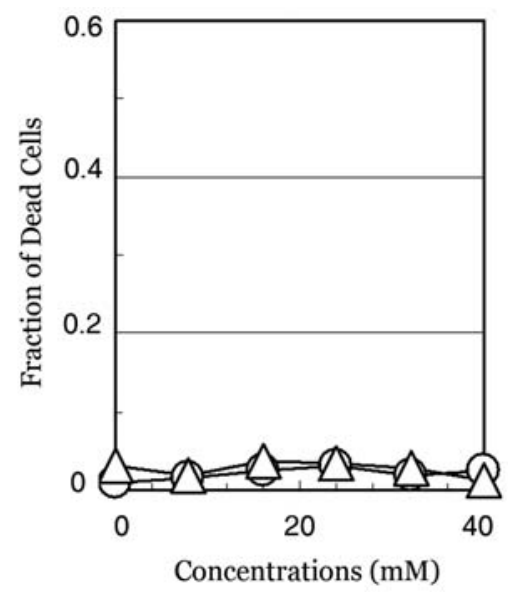

Figure 2. Effect of arginine on the viability of HEp-2 cells. Confluent monolayers of HEp-2 cells were incubated in MEM containing $0.1 \%$ BSA containing various concentrations of arginine or sodium chloride at $37^{\circ} \mathrm{C}$. After $21 \mathrm{~h}$ of incubation, the cells were trypsinized to obtain a single cell suspension and the numbers of the living and the dead cells in each culture were determined by a dye-exclusion test with trypan blue. Arginine $(\odot)$; sodium chloride $(\triangle)$.

When HEp-2 cells were incubated with arginine, both rounding and shrinkage of the cells were observed, but without cell detachment from the dishes; even at the concentration of $20 \mathrm{mM}$, arginine induced CPE in a small but significant population of the cells. The CPE was enhanced with increasing concentrations of arginine; namely more cells suffered CPE.

To examine quantitatively the cytotoxic effect of arginine on HEp- 2 cells, the number of dead and living cells upon incubation of HEp-2 cells with arginine for $21 \mathrm{~h}$, was determined as a function of arginine concentration by a dyeexclusion test (Fig. 2). Cell death was only 1-3\% of the total cell population independently of arginine concentrations 


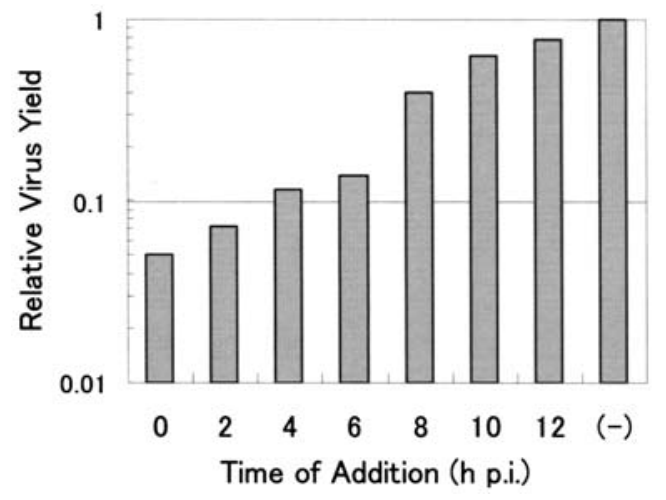

Figure 3. Effect of 'time of addition' of arginine on the final virus yield. HEp-2 cells, infected with HSV-1 at a MOI of 15, were incubated in MEM containing $0.1 \%$ BSA. At various times after the infection, arginine was added to the culture medium at the final concentration of $30 \mathrm{mM}$. At $26 \mathrm{~h}$ p.i., the amount of total progeny virus in each culture was assayed. Relative virus yields in the reagent-treated cultures to that in the untreated culture were calculated.

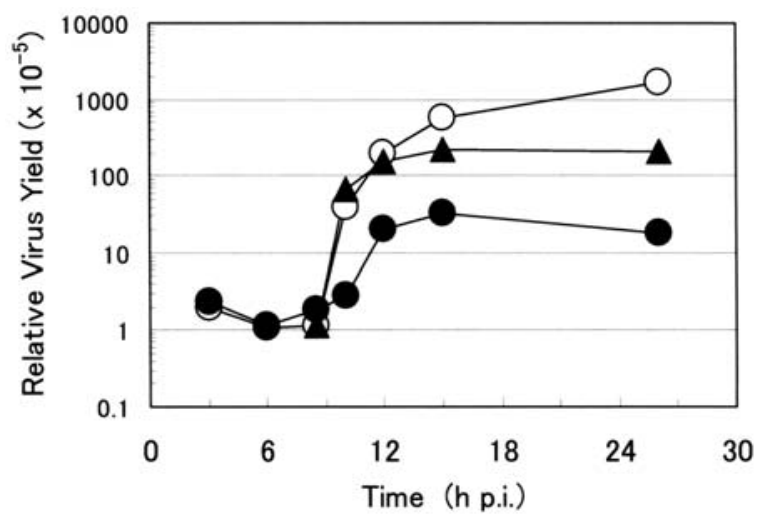

Figure 4. One-step growth curve of HSV-1 in the presence or absence of arginine. Confluent monolayers of HEp-2 cells were infected with HSV-1 at a MOI of 15 . The infected cells were incubated at $37^{\circ} \mathrm{C}$ in MEM containing $0.1 \%$ BSA $(\circ)$ or in the medium additionally containing $30 \mathrm{mM}$ arginine $(\bullet)$ At $8.5 \mathrm{~h}$ p.i., a group of cultures received $30 \mathrm{mM}$ arginine in the culture medium $(\mathbf{\Lambda})$. At the indicated time, the amounts of total progeny viruses in the cultures were determined.

(0-40 mM). As described above, a much larger cell population underwent $\mathrm{CPE}$ upon incubation with arginine, meaning that CPE does not necessarily cause cell death, hence the cells suffering arginine-induced CPE are still viable with functionally normal plasma membrane integrity. This in turn suggests that the observed decrease in the virus yield by arginine is unlikely due to the non-specific degeneration (cell death) of the infected cells by the cytotoxicity of arginine. This suggestion is further confirmed by the observations that HSV-1 infection completely abolished arginine-induced CPE: namely, arginine does not induce CPE in HEp-2 cells infected with $\mathrm{HSV}-1$, probably due to the expression of anti-apoptotic genes of HSV-1 in the host cells (11). It can be concluded that the observed antiviral activity of arginine is not due to its CPE, at least for HSV-1 and that other factors are involved.

As arginine is a monovalent ion, its addition increases the ionic strength of the cell culture media. The contribution of

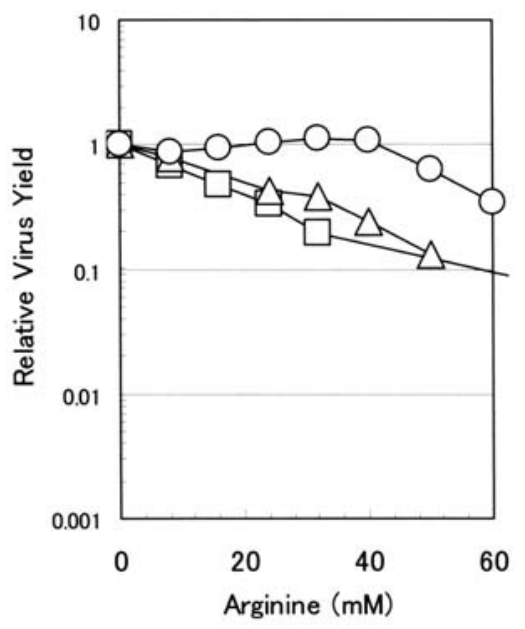

Figure 5. Effect of arginine on the yield of RNA viruses. Confluent monolayers of HEp-2 (for VSV and poliovirus) or MDCK (for influenza virus) cells were infected with VSV, poliovirus or influenza virus at a MOI of 10 . The infected cells were incubated at $37^{\circ} \mathrm{C}$ (for influenza virus and VSV) or $35.5^{\circ} \mathrm{C}$ (for poliovirus) for $\sim 14 \mathrm{~h}$ in MEM containing $0.1 \%$ BSA and the indicated concentrations of arginine. For influenza virus, acetylated trypsin was additionally supplemented to the culture medium. The amounts of progeny viruses in the culture media were determined as described in Materials and methods and were normalized to the virus yield in the absence of the reagent. VSV $(\odot)$; influenza virus $(\triangle)$; poliovirus $(\square)$.

ionic strength to the cell death and CPE on HEp-2 cells was examined using $\mathrm{NaCl}$. The results, shown in Fig. 2, clearly indicate that $\mathrm{NaCl}$ also caused a $1-3 \%$ cell death within the same concentration range. Therefore, the observed cell death with arginine was most likely due to the non-specific effect of ionic strength. However, there was no $\mathrm{CPE}$ by $\mathrm{NaCl}$ on HEp-2 cells within this concentration range in contrast to the notable CPE with arginine. Thus, such a small increase in ionic strength does not cause significant CPE. These findings indicate that arginine has a unique property that is responsible for the observed CPE. Such a property may be related to its effects on the host cell-virus interaction.

Understanding the step in the viral multiplication cycle that is most sensitive to arginine treatment should give an insight into the mechanism of the antiviral activity of arginine. To elucidate this, arginine was added to the culture medium of the infected cells at various times post-infection (p.i) and the progeny virus was harvested at the end of virus multiplication (26 h p.i). As shown in Fig. 3, results of this 'time of addition' study showed that arginine completely inhibited the formation of progeny virus by the addition before and at $6 \mathrm{~h}$ p.i. when the viral DNA replication is completed in the HSV-1-infected cells (12). These results indicate that the formation of progeny virus is still sensitive to the reagent even after the completion of viral DNA replication. The effect of arginine was greatly reduced when added at $8 \mathrm{~h}$ p.i., suggesting that there is no critical reagentsensitive step in the late stage ( $8 \mathrm{~h}$ p.i. or later) of the virus multiplication.

Fig. 4 shows a one-step growth curve of HSV-1 in the presence or absence of $30 \mathrm{mM}$ arginine; arginine was added at 0 or $8 \mathrm{~h}$ p.i. When added at $0 \mathrm{~h}$ p.i., i) the onset of infectious progeny virus production was delayed for $\sim 2 \mathrm{~h}$, ii) the rate 
of formation of progeny infectious virus was decreased and iii) the final yield of progeny virus decreased to $1 \%$. This is consistent with the arginine-sensitive step at $\sim 6 \mathrm{~h}$ p.i. However, when added at $8 \mathrm{~h}$ p.i., the virus grew normally for $\sim 4 \mathrm{~h}$ following the addition of arginine. Again, this is consistent with the above finding that there is no critical arginine-sensitive step in the late stage of the infection. This also confirms that arginine does not directly interfere with the formation of progeny infectious virus. Nevertheless, the final yield was lower, probably due to a non-specific physiological alteration in the infected cells by the reagent.

The antiviral activity of arginine was also examined for RNA viruses of three different families, i.e., influenza virus (Orthomyxoviridae family), poliovirus (Picornaviridae family) and VSV (Rhabdoviridae family). Both influenza virus and VSV are enveloped viruses whereas poliovirus is a nonenveloped virus. Influenza virus was grown on MDCK cells, while poliovirus and VSV were grown on HEp-2 cells as for $\mathrm{HSV}-1$. Arginine concentration-dependently inhibited the multiplication of influenza virus and poliovirus, as shown in Fig. 5, although the sensitivities of these two RNA viruses to arginine were much weaker than the response of HSV-1. The virus yields decreased only by 10 -fold for both viruses at $50 \mathrm{mM}$ arginine. As both poliovirus and HSV-1 were grown in HEp-2 cells, the observed lower sensitivity of poliovirus to arginine most likely reflects the intrinsic property of the poliovirus. Lower sensitivity of influenza virus could be due to the different host cells used and the virus nature itself.

On the other hand, arginine was ineffective against VSV up to $40 \mathrm{mM}$ (Fig. 5). A small inhibition was observed at higher arginine concentrations $(50-60 \mathrm{mM})$ and may be ascribed to a secondary result of non-specific cytopathic effects of arginine, e.g., high osmotic pressure, which possibly caused degeneration of the infected cells. Although HEp-2 cells were used for HSV-1, poliovirus and VSV, only VSV exhibited a strong resistance to arginine. This indicates that the resistance of VSV is most likely due to the intrinsic property of the virus.

\section{Discussion}

The present study clearly showed the inhibition of the multiplication of HSV-1 by arginine. The collective findings concerning the effects of arginine on the virus yields (Fig. 1), 'time-ofaddition' experiments (Fig. 3) and the one-step growth curve (Fig. 4) clearly indicated that this amino acid effectively inhibited the multiplication of HSV-1 in the infected cells. The primary reagent-sensitive step in the virus multiplication is the step(s) prior to the maturation (assembly) of infectious progeny virus, as is evident from the following results. First, while the addition of the reagent at $6 \mathrm{~h}$ p.i. completely suppressed the formation of progeny virus, the addition at $8 \mathrm{~h}$ p.i. did so inefficiently (Fig. 3). Second, the addition of the reagent at $8 \mathrm{~h}$ p.i. allowed the normal progeny virus formation in the subsequent $4 \mathrm{~h}$, indicating that the formation of progeny virus itself is not directly interfered with by arginine. Previously, we revealed that in HSV-1-infected cells, viral DNA rapidly replicates between 3 and $6 \mathrm{~h}$ p.i. and the formation of both the nucleocapsids and the progeny infectious viral particles gradually takes place from 5 to $12 \mathrm{~h}$ p.i. under these conditions (12). The results in Fig. 3 clearly indicate that even after the completion of viral DNA replication (at $6 \mathrm{~h}$ p.i.) the addition of arginine can completely abolish the formation of the infectious progeny virus.

One of the mechanisms of antiviral agents is the selective acceleration of cell death of virus-infected cells by the antiviral agent $(13,14)$. However, arginine showed no acceleration of cell death of the HSV-1 infected cells, demonstrating that a significant decrease in the progeny virus production by arginine is not due to its selected cytopathic effects on the infected cell. It is not clear at this stage how arginine suppresses the progeny virus production. It has been suggested that arginine affects virus growth through its effect on nitric oxide synthase $(15,16)$, although the involvement of this enzyme and hence nitric oxide in the observed anti-HSV-1 activity of arginine is still unclear.

Whatever the mechanism involved, arginine does have antiviral activities against several DNA and RNA viruses (Figs. 1 and 5), in particular against HSV-1 at a moderate concentration. Yet, it would be difficult to achieve the required high serum arginine concentration needed to use arginine against systemic infections. However, such concentration can be readily achieved for topical applications. HSV-1 causes serious topical diseases and hence antiviral activities of arginine may find applications in these areas $(2,17)$.

\section{Acknowledgements}

We thank Dr Daisuke Ejima (Ajinomoto, Japan) for his helpful discussion and Ms. Misao Uozaki and Dr Yukiko Katsuyama for their technical assistance. This work is supported in part by a research grant from Ajinomoto Co. Inc.

\section{References}

1. Cowan MM: Plant products as antimicrobial agents. Clin Microbiol Rev 12: 564-582, 1999.

2. Isaacs CE, Wen GY, Xu W, Jia JH, Rohan L, Corbo C, Di Maggio V, Jenkins EC Jr and Hiller S: Epigallocatechin gallate inactivates clinical isolates of herpes simplex virus. Antimicrob Agents Chemother 52: 962-970, 2008.

3. Katsuyama Y, Yamasaki H, Tsujimoto K, Koyama AH, Ejima D and Arakawa T: Butyroyl-arginine as a potent virus inactivation agent. Int J Pharm 361: 92-98, 2008.

4. Yamasaki H, Tsujimoto K, Koyama AH, Ejima D and Arakawa T: Arginine facilitates inactivation of enveloped viruses. J Pharm Sci 97: 3067-3073, 2008.

5. Utsunomiya H, Ichinose M, Uozaki M, Tsujimoto K, Yamasaki H and Koyama AH: Antiviral activities of coffee extracts in vitro. Food Chem Toxicol 46: 1919-1924, 2008.

6. Brorson K, Krejci S, Lee K, Hamilton E, Stein K and Xu Y: Bracketed generic inactivation of rodent retroviruses by low $\mathrm{pH}$ treatment for monoclonal antibodies and recombinant proteins. Biotechnol Bioeng 82: 321-329, 2003.

7. Koyama $\mathrm{AH}$ and Uchida T: The effect of ammonium chloride on the multiplication of herpes simplex virus type 1 in Vero cells. Virus Res 13: 271-282, 1989.

8. Koyama AH: Induction of apoptotic DNA fragmentation by the infection of vesicular stomatitis virus. Virus Res 37: 285-290, 1995.

9. Koyama AH, Irie H, Ueno F, Ogawa M, Nomoto A and Adachi A: Suppression of apoptotic and necrotic cell death by poliovirus. J Gen Virol 82: 2965-2972, 2001.

10. Kurokawa M, Koyama AH, Yasuoka S and Adachi A: Influenza virus overcomes apoptosis by rapid multiplication. Int J Mol Med 3: 527-530, 1999.

11. Koyama AH and Miwa Y: Suppression of apoptotic DNA fragmentation in herpes simplex virus type 1-infected cells. J Virol 71: 2567-2571, 1997. 
12. Koyama AH and Uchida T: Quantitative studies on the maturation process of herpes simplex virus type 1 in Vero cells. Virus Res 10: 281-286, 1988

13. Koyama AH, Arakawa T and Adachi A: Acceleration of virusinduced apoptosis by tumor necrosis factor. FEBS Lett 426 : $179-182,1998$

14. Koyama AH, Fukumori T, Fujita T, Irie $\mathrm{H}$ and Adachi $\mathrm{A}$ : Physiological significance of apoptosis in animal virus infection. Microbes Infect 2: 1111-1117, 2000.
15. Takada H, Kishimoto C, Hiraoka Y and Ochiai H: Oral Larginine prevents murine coxsackievirus B3 myocarditis. Int J Cardiol 86: 272-279, 2002.

16. Agawa $\mathrm{H}$, Ikuta $\mathrm{K}$, Minamiyama $\mathrm{Y}$, Inoue $\mathrm{M}$ and Sairenji T: Down-regulation of spontaneous Epstein-Barr virus reactivation in the P3HR-1 cell line by L-arginine. Virology 304: 114-124, 2002.

17. Wu JJ, Pang KR, Huang DB and Tyring SK: Advances in antiviral therapy. Dermatol Clin 23: 313-322, 2005. 